Itinéraires Itinéraires

Littérature, textes, cultures

2017-1 | 2018

Biographie et fiction

\title{
Biographie et fiction : Les Vies imaginaires de Walter Pater
}

Biography and Fiction: Imaginary Portraits, by Walter Pater

\section{Alexandre Bies}

\section{(2) OpenEdition}

\section{Journals}

Édition électronique

URL : http://journals.openedition.org/itineraires/3751

DOI : 10.4000/itineraires. 3751

ISSN : 2427-920X

Éditeur

Pléiade

Référence électronique

Alexandre Bies, «Biographie et fiction : Les Vies imaginaires de Walter Pater », Itinéraires [En ligne], 2017-1 | 2018, mis en ligne le 15 février 2018, consulté le 30 septembre 2020. URL : http://

journals.openedition.org/itineraires/3751 ; DOI : https://doi.org/10.4000/itineraires.3751

Ce document a été généré automatiquement le 30 septembre 2020

\section{(c) (i) () $\Theta$}

Itinéraires est mis à disposition selon les termes de la licence Creative Commons Attribution - Pas d'Utilisation Commerciale - Pas de Modification 4.0 International. 


\title{
Biographie et fiction : Les Vies imaginaires de Walter Pater
}

\author{
Biography and Fiction: Imaginary Portraits, by Walter Pater
}

\section{Alexandre Bies}

1 Dans l'introduction qui précède sa traduction des Studies in the History of the Renaissance, Frédéric Roger-Cornaz rappelle une anecdote qui montre d'emblée de quelle manière Walter Pater propose une conception originale de la biographie, qui n'est pas une restitution des faits mais se tisse d'expériences imaginaires, ainsi que le manifeste la conviction qui fut la sienne de jouir d'une généalogie illustre :

Walter Pater naquit en 1839 à Shadwell d'une famille de bourgeoisie honorable. Le nom de Pater n'est pas commun en Angleterre; et l'écrivain qui a si bien parlé de Watteau dans un de ses Portraits imaginaires, se plut toujours à penser qu'il était de la même race que Jean-Baptiste Pater ${ }^{1}$, le frivole et brillant élève du peintre de

Valenciennes. (Roger-Cornaz $1917: 5$ )

2 Cette filiation supposée est d'autant plus significative que, parmi les Portraits imaginaires ${ }^{2}$ dont il fut l'auteur, l'un d'eux fut consacré à Watteau, comme s'il s'agissait de prolonger cette croyance, à laquelle le récit viendrait donner plus de consistance. En effet, cette conception de l'identité s'inscrit pour Pater, dans l'héritage de la philosophie d'Héraclite et d'un monde en perpétuel mouvement, dans lequel rien de stable ne saurait exister, qu'il s'agisse de la nature ou de l'individu ${ }^{3}$. Cette relecture d'une pensée antique du mouvement trouve de nombreux échos dans des préoccupations contemporaines ${ }^{4}$, et notamment dans les récentes découvertes scientifiques qui conduisent à adopter, suite aux écrits de Darwin, un modèle de pensée qui emprunte pour une large part à un paradigme biologique : «The origins of Pater's Aesthetic hero lay in the new science being proclaimed by men such as Charles Darwin, Thomas Henry Huxley, John Tyndall, John Stuart Mill, and Herbert Spencer; namely, the new spirit of relativism, of flux » (Monsman $1977: 10)^{5}$.

3 Ainsi, dénué de toute identité qui subsisterait au-delà des modifications continuelles qu'impose le cours du temps, l'individu n'existe que dans l'instant et à travers ses expériences. Son lot de sensations, loin de lui attribuer une unité, est le lieu d'une 
irréductible diversité qui ouvre, par des voies nombreuses, à autant de vies possibles. Pater inverse le rapport habituel selon lequel la biographie serait le récit d'une vie constituée pour faire du récit la forme même d'une existence qui se trouve alors tissée de fiction. Si seule compte la sensation, alors l'imaginaire ou le rêve, l'approfondissement d'une sensation diffuse auront au moins autant de valeur que ce qu'on considère d'ordinaire comme des faits établis. Une telle conception influencera l'œuvre de Pater, non seulement dans le choix de personnages, en tant qu'ils appartiennent à des moments historiques de transition ${ }^{6}$, mais plus encore par son style et une manière d'écrire qui ne cesse de se reprendre elle-même, de se corriger pour parvenir à saisir toujours mieux une réalité en train de se faire :

Le style d'un Pater [...] n'est pas un style d'action, mais de contemplation, d'attente, d'évasion. Il se rapproche non de l'architecture mais de la musique (ce fut précisément Pater qui reprit de la philosophie allemande l'idée que tout art aspire aux conditions de la musique) : un thème peut se développer en variations infinies, souvent liées entre elles de façon si subtile que le rapport se perd. (Praz 1990 : 40)

Ce style atypique témoignerait à lui seul d'une certaine manière d'être ou d'une conduite esthétique qui sous-tend par sa forme même une représentation du monde dans la mesure où, ainsi que le souligne Marielle Macé (2011 : 15), « toute configuration littéraire indique ainsi quelque chose comme une piste à suivre, un phrasé dans l'existant ». On pourra cependant se demander si c'est là la compensation qu'offre la fiction à une existence retirée du monde, consacrée tout entière à la lecture, et comparable pour celui qui avait pour jeu d'enfant d'imiter la liturgie catholique ${ }^{7}$, à une vie monacale. ${ }^{8}$. En ce sens, l'impossible biographie d'une existence tout entière réduite à l'étude, laisserait la place à l'imaginaire. Ce serait à tort qu'on ferait du souci biographique chez Pater le pendant d'une existence qui n'aurait pas été véritablement vécue, ainsi que semble le suggérer Wilde, lorsqu'il disait à propos de Pater : «A-t-il jamais été vivant? » (cité par Ellmann 1994 : 71). Quelle confiance accorder dès lors à la compréhension de la vie humaine chez un tel auteur, dont la vie ne serait pas conforme à son exigence de mener une existence intense et passionnée ?

5 Or, n'est-ce pas l'isolement lui-même qui rend possible de saisir avec une plus grande acuité la physionomie des âmes ? Ce « reclus volontaire » (Neel 1930 : XIII) ne conserve ses distances avec le monde qu'afin d'en restituer mieux les images et la peinture sensible, comme si l'ascèse seule devait permettre ce recentrement sur soi par lequel il lui est possible de ressaisir avec plus de justesse ses propres impressions et de les déployer sous la forme de nombreux destins. Pater serait alors semblable à l'un de ses personnages, Antoine Watteau, dont la qualité des œuvres tient à l'absence de sympathie qu'il porte à son objet, autrement dit à sa capacité à se retirer du monde pour n'être qu'un simple spectateur capable de saisir les secrets de ce qu'il représente :

Antony Watteau reproduces that gallant world, those patched and powdered ladies and fine cavaliers, so much to its own satisfaction, partly because he despises it; if this be a possible condition of excellent artistic production. (IP : 33) ${ }^{9}$

6 Après avoir été frappé d'interdiction, tant par «la doxa du Contre Sainte-Beuve » (Boyer-Weinmann 2004 : 299) que par la mise à distance du sujet par le structuralisme, il convient pourtant de ne pas céder, face à une prolifération des publications biographiques contemporaines, à l'impensé d'une injonction à se réaliser soi-même réduite à un simple slogan. La récurrence de l'élément biographique chez Pater trouve son explication dans le souci profondément éthique qui anime ses œuvres de fiction aussi bien que ses essais, qui partagent tous une même interrogation, à savoir: 
comment vivre ${ }^{10}$ ? Ainsi, Pater anticipe sur des préoccupations contemporaines, puisque, depuis le "tournant éthique " (Korthals Altes 1999), la littérature semble être devenue le lieu privilégié pour interroger nos formes de vie ${ }^{11}$.

\section{L'élément biographique dans la critique patérienne}

7 Il nous faut souligner d'emblée l'importance du souci biographique chez Pater, qui met en échec les lignes de partage habituelles pour lui préférer un « espace d'ambiguïté ou de transgression générique » (Gefen 2002: 3), dans la mesure où ses essais aussi bien que ses œuvres de fiction semblent avoir en commun, non seulement l'intérêt pour le récit d'une vie humaine, mais plus encore la forme du portrait :

Les «portraits imaginaires" sont le prolongement des essais critiques de Pater qui placent l'œuvre d'art, l'idée philosophique ou le livre au centre d'un environnement culturel derrière lequel il devine le pouvoir d'une personne. La réflexion naît donc d'une véritable rencontre entre deux subjectivités, celle de l'interprète et celle de l'individu pressenti, ce qui fait de son récit, comme le souligne Germain d'Hangest, une forme d'autoportrait. (Lambert-Charbonnier $2004: 9$ )

En effet, Walter Pater est l'auteur de nombreux Portraits imaginaires, qui sont des récits d'esthètes ou d'artistes à différentes époques, mais c'est dès ses écrits critiques, rassemblés dans La Renaissance, que se dégage le motif du portrait et de la vie imaginaire. Il se propose, en effet, moins une étude précise de l'œuvre qu'une tentative de dégager un caractère ou un tempérament, autrement dit une personnalité qui préside à la création. Sans relever explicitement d'un projet biographique, s'y dessine déjà une approche qui serait celle du récit d'une vie ${ }^{12}$. Dans le cas de Giorgione, il parle par exemple d'une tension poétique résultant d'une certaine manière de vivre (« While he interfuses his painted work with a high-strung sort of poetry, caught directly from a singularly rich and high-strung sort of life ", Pater 1873: 142), ou encore, il évoque dans le cas de Luca Della Robbia, une sculpture manifestant la profondeur de son âme (" that profound expressiveness, that intimate impress of an indwelling soul, which is the peculiar fascination of the art of Italy in that century ", Pater $1873: 63$ ). Et à propos de Vinci, il esquisse le portrait rapide d'une jeunesse passionnée :

We see him in his boyhood fascinating all men by his beauty, improvising music and songs, buying the caged birds and setting them free, as he walked the streets of Florence, fond of odd bright dresses and spirited horses. (Pater [1873] $1912: 10$ )

L'approche biographique de la critique, qui ferait l'économie de la rigueur historique au profit de la qualité du portrait annonce déjà l'émergence d'un genre littéraire. Cela s'explique par l'importance accordée par Pater à la subjectivité. Qu'il s'agisse de celle du créateur ou du critique, l'univers de Pater n'est plus un monde objectif et fondé sur la solidité des lois scientifiques. Bien au contraire, seule a de la valeur l'expérience que fait un individu, dans un monde où tout est indifféremment soumis à un régime d'existence éphémère : «inconstant modes or fashions" (Pater [1873] 1912: 233). L'analyse de toute chose adoptant le modèle chimique pour en énumérer les composants (phosphore, calcium, fibrilles délicates), Pater peut affirmer que seules nos sensations relèvent d'une certaine vérité. Il fait de cette découverte d'une identité mobile et de cette compréhension du mouvement qui affecte jusqu'à la conscience, l'intuition fondatrice de la culture européenne naissant avec la philosophie grecque :

From the lifeless background of an unprogressive world-Egypt, Syria, frozen Scythia-a world in which the unconscious social aggregate had been everything, 
the conscious individual, his capacity and rights, almost nothing, the Greek had stepped forth, like the young prince in the fable, to set things going. (Pater [1893] $1910: 21)$

10 Cela doit donc déterminer la méthode propre au critique et attirer son attention aussi bien sur ce qui se manifeste de l'individualité d'un artiste dans son travail que sur les impressions propres au critique ou au spectateur, au contact de l'œuvre. La contestation de toute définition abstraite de la beauté dans l'introduction de La Renaissance expose cette manière originale pour appréhender la relativité du beau. Puisqu'il n'est plus question de déterminer des éléments objectifs définissant la beauté, il convient alors pour le critique d'être avant tout attentif aux émotions ressenties, à leurs qualités, leur profondeur, et de s'attacher, comme l'écrit Pater, à les démêler finement (« to know one's own impression as it really is, to discriminate it, to realise it distinctly ", Pater [1873] 1912 : VIII). Il s'agit d'évaluer l'écho que trouve l'œuvre en soi :

What is this song or picture, this engaging personality presented in life or in a book, to me? What effect does it really produce on me? Does it give me pleasure? and if so, what sort or degree of pleasure? How is my nature modified by its presence, and under its influence? (Pater [1873] 1912 : VIII)

11 Une telle méthode rend inutiles toutes les préoccupations métaphysiques, et recentre le sujet sur l'expérience qu'il fait à l'occasion de certains objets considérés comme esthétiques et qui ne se limitent pas aux œuvres d'art mais comprend les belles formes de la nature et de la vie humaine. Puisque la question centrale du travail du critique est de savoir, à propos de ces objets, les effets qu'ils ont sur soi, il apparaît que l'approfondissement de soi est une condition de possibilité du bon critique, dont le tempérament doit le rendre apte à s'émouvoir profondément des beaux objets et qu'il devra exercer par la fréquentation des beautés artistiques et naturelles aussi bien que par ce travail précis d'examen des effets en lui. Dans le cas de Winckelmann, Pater va jusqu'à affirmer que son appréciation de l'art est le résultat de sa compagnie de beaux jeunes gens, si bien que sa qualité d'historien de l'art tient avant tout à son enthousiasme et son désir, à sa capacité à saisir la beauté des jeunes garçons qui le rend sensible à la sculpture grecque et apte à la comprendre :

That his affinity with Hellenism was not merely intellectual, that the subtler threads of temperament were inwoven in it, is proved by his romantic, fervent friendships with young men. He has known, he says, many young men more beautiful than Guido's archangel. (Pater [1873] $1912: 191$ )

La méthode propre à Winckelmann qui est intuitive, fondée sur cette attention au réel et non sur des compétences discursives, résulte de sa vie même, de son caractère et des expériences qui ont façonné sa sensibilité, au point d'engendrer en lui des instruments propres à la saisir: "that world in which others had moved with so much embarrassment, seems to call out in Winckelmann new senses fitted to deal with it » (Pater 1873 : 194). Pour ces mêmes raisons, l'approche critique de Pater pourra être contestée dans la mesure où ses interprétations seraient trop subjectives, privilégiant cette compréhension personnelle au détriment d'une lecture objective. Celui qui considère qu'il n'existe de réalité que saisie par le prisme d'une psychologie singulière, se voit alors accusé de pervertir l'histoire et de falsifier la réalité (More 1913). Or, dans le traité qu'il consacre à la question du style, il défend les qualités de la prose et l'idée que toute écriture traduit une individualité. Il conteste ainsi la conception d'une écriture neutre et objective, allant jusqu'à affirmer que même la rigueur des sciences ne saurait y échapper totalement, et que des activités qu'on croit n'être pas artistiques 
portent tout de même la marque du style ${ }^{13}$. C'est notamment le cas de l'histoire où la sélection des faits sera déterminée par un état d'âme, à la façon dont Tite-Live, Tacite ou Michelet ont modifié le passé en y introduisant leur propre sensibilité :

Si son esthétique, avec les années, s'assouplit graduellement de plusieurs nuances nouvelles, et si même, dans le temps qu'il écrivait La Renaissance, des perspectives diverses le sollicitèrent tour à tour, il est une idée fondamentale que l'ensemble de son œuvre ne cessa point d'affirmer avec force, c'est que l'art, quoi qu'il puisse par ailleurs nous offrir, est d'abord l'expression de l'artiste lui-même dans son individuelle singularité. [...]. C'est en effet dans ce lien mystérieux des œuvres avec la personnalité dont elles sont issues que réside en fin de compte pour lui leur suprême, leur inépuisable intérêt; et toutes, de quelque côté qu'il se tourne, lui semblent confirmer avec éclat cette vérité centrale. (Hangest 1961, $1: 133-134)^{14}$

En effet, l'ambition de Pater est sans doute, plutôt que de restituer avec rigueur la doctrine elle-même, de percevoir l'homme lui-même : "the spirit of the man: that is the thing for him! » (Johnson $1911: 1$ ). En ce sens, les études consacrées à des artistes participent déjà de l'élaboration de vies imaginaires, en déplaçant l'enjeu d'une critique érudite vers une critique créatrice, consistant à « faire du récit d'une vie humaine non un savoir, mais l'occasion d'un jeu littéraire, d'une rêverie ou d'une méditation » (Gefen 2014 : 7). De cette façon, l'ensemble des écrits de Pater, les textes théoriques ou les essais, repose sur le principe méthodologique d'une individualité irréductible, qui ne saurait être remplacée par aucune autre et qui interdit de la négliger dans l'étude d'une œuvre. En dehors des artistes dont il étudie le tempérament dans La Renaissance, cet aspect est tout aussi prédominant dans les conférences qu'il consacre à la philosophie antique dans Platon et le platonisme. Il va jusqu'à en dégager un conseil pratique à l'adresse des étudiants dans la manière d'aborder un tel exercice :

Better still, as in reading Hamlet or The Divine Comedy, so in reading The Republic, to watch, for its dramatic interest, the spectacle of a powerful, of a sovereign intellect, translating itself, amid a complex group of conditions which can never in the nature of things occur again, at once pliant and resistant to them, into a great literary monument. (Pater [1893] $1910: 23$ )

Dans l'évocation que fait Pater, sans distinction entre des œuvres philosophiques et littéraires, il met en avant l'importance qu'a la personnalité dans le processus de création. Insistant sur l'importance de resituer un auteur et une pensée dans un contexte dans la mesure où ils résultent de circonstances historiques particulières, Pater propose ainsi deux principes explicatifs de toute œuvre: la détermination d'une époque et la capacité d'une personnalité à se dresser contre elle à partir de son propre fond:

There is always also, as if acting from the opposite side, the comparatively inexplicable force of a personality, resistant to, while it is moulded by, them. [...] We touch what is unique in the individual genius which contrived after all, by force of will, to have its own masterful way with that environment. If in reading Plato, for instance, the philosophic student has to re-construct for himself, as far as possible, the general character of an age he must also, so far as he may, reproduce the portrait of a person. (Pater [1893] 1910 : 124-125)

Or, si les articles et les essais visent à dégager une individualité créatrice, le brouillage des genres joue également en sens inverse, dans la mesure où les Portraits, bien qu'il s'agisse de récits de fiction, ont pour vocation de rendre sensibles certaines abstractions. Ce projet de pensées incarnées marquera d'ailleurs un point de rupture avec Marcel Schwob, pourtant largement inspiré par l'œuvre de Pater, en ce qu'il se proposera d'entreprendre le récit d'individualités absolument dégagées de tout type. 
En effet, selon lui, l'écrivain à la différence de l'historien devrait se méfier de toute forme de généralisation, ne se consacrant qu'à l'unique et à l'individuel selon un art du déclassement plutôt que de la catégorisation. S'opposant ainsi à des figures qui ne seraient qu'idées animées sous des traits humains, Marcel Schwob se donne comme exigence de l'écriture biographique "de différencier infiniment l'aspect de deux philosophes qui ont inventé à peu près la même métaphysique »(Schwob 1896:511) ${ }^{15}$.

Or, qu'il s'agisse de la conception récurrente du rôle de l'art dans l'existence, ou de philosophies mises en mouvement sous les traits d'une figure humaine, on retrouve bien l'exigence de Pater de ne jamais s'en remettre à un exposé abstrait et d'employer la fiction comme une manière d'exposer des doctrines sous la forme d'une personnalité ${ }^{16}$. Il l'avoue lui-même en conclusion du portrait consacré au duc Carl de Rosenmold, qui devait incarner la naissance de l'esprit des Lumières en Allemagne, comme une renaissance de l'esprit au contact des arts :

The Enlightening, the Aufklärung, according to the aspiration of Duke Carl, was effected by other hands; Lessing and Herder, brilliant precursors of the age of genius which centered in Goethe, coming well within the natural limits of Carl's lifetime. As precursors Goethe gratefully recognised them, and understood that there had been a thousand others, looking forward to a new era in German literature with the desire which is in some sort a "forecast of capacity," awakening each other to the permanent reality of a poetic ideal in human life, slowly forming that public consciousness to which Goethe actually addressed himself. It is their aspirations I have tried to embody in the portrait of Carl. (IP : 152) ${ }^{17}$

\section{Walter Pater et ses Portraits imaginaires}

17 S'il existe avant Pater des Vies parallèles et des récits de vie de héros ou de grands hommes, ou parmi ses contemporains les Portraits littéraires de Sainte-Beuve ${ }^{18}$, son influence semble remarquable sur l'invention d'un genre littéraire nouveau qui marque la modernité avec Schwob, mais plus largement, dans l'attention accordée à la vie intérieure et à ses détails chez Joyce ou Woolf (Beckson et Munro 1970), et jusqu'à la postmodernité chez Robbe-Grillet ou Leiris (Monsman 1980 : 5). En effet, si Pater porte beaucoup d'attention aux personnalités qu'il examine dans ses essais, c'est bien sous la forme de la fiction, et avec la liberté que lui confèrent les Portraits imaginaires, qu'il déploie totalement le projet éthique qui sous-tend son écriture dans l'invention qu'il propose de destinées humaines ${ }^{19}$. Celles-ci cependant viennent s'alimenter aux souvenirs de leur auteur et empruntent ainsi à l'autobiographie imaginaire ou à la biofiction (Buisine 1991). Voici comment il définit lui-même son projet à l'occasion de la publication du premier de ces Portraits :

I send you by this post a M.S. entitled The House and the Child, and should be pleased if you should like to have it for Macmillans' Magazine. It is not, as you may perhaps fancy, the first part of a work of fiction, but is meant to be complete in itself; though the first of a series, as I hope, with some real kind of sequence in them, and which I should be glad to send to you. I call the M.S. a portrait, and mean readers, as they might do on seeing a portrait, to begin speculating-what came of him? (Pater 1970 : 29-30)

18 Les Portraits sont bien des récits de vie, exposant les origines de leurs héros, et racontant leurs expériences, leur itinéraire, et ce jusqu'à leur mort. Ils rendent compte de leurs ascendances à travers leur nom comme dans le cas du peintre Watteau ${ }^{20}$ au 
patronyme évocateur, ou recourent de manière plus explicite à la légende, comme dans le cas de Denys l'Auxerrois, Dionysos ressuscité dans le Moyen Âge français :

What follows is a quaint legend, with detail enough, of such a return of a golden or poetically-gilded age (a denizen of old Greece itself actually finding his way back again among men) as it happened in an ancient town of medieval France. (IP : 47) existence plus conforme à leurs attentes, à l'instar du duc de Rosenmold, aspirant à une rénovation de l'art dans la sombre Allemagne par l'intermédiaire de la lumière de la Grèce et de l'Italie et désire profondément se découvrir des ancêtres dans la Méditerranée :

At times the fancy came that he must really belong by descent to a southern race, [...]. The aged ministers of heraldry were set to work (actually prolonging their days by an unexpected revival of interest in their too well-worn function) at the search for some obscure rivulet of Greek descent-later Byzantine Greek, perhaps,-in the Rosenmold genealogy. (IP : 133-134)

en des documents destinés à établir sa généalogie illustre le travail du biographe même, le travail d'érudition de Pater, rédigeant des vies inspirées d'existences avérées, ou ne faisant que croiser des hommes ayant existé et assistant au spectacle d'une histoire avérée ${ }^{21}$. Plus que tout autre, le portrait consacré à Denys l'Auxerrois insiste sur le motif du vestige ou de la relique ${ }^{22}$ comme source d'inspiration, allant chercher à la source de la mémoire, ici collective, pour réveiller des sensations enfouies et oubliées qui portent en elles le germe d'une création et d'un récit nouveau. Les frontières se brouillent alors entre la légende et l'histoire, autrement dit en un temps oublié qui échappe à la question de l'authenticité pour confondre la fiction et la vérité, en empruntant à l'exactitude des ruines pour ouvrir à la rêverie où se déploie le souvenir des vies antérieures : " a physical cause might lie beneath this strange restlessness, like the imperfect reminiscence of something that had passed in earlier life » (IP : 133).

Le portrait «Un prince des peintres de cour» consacré au peintre Antoine Watteau occupe une place singulière dans les Portraits imaginaires dans la mesure où il échappe au modèle habituel construit autour d'un narrateur. En effet, dans celui-ci, Pater adopte le dispositif littéraire du journal, rédigé par une jeune fille de Valenciennes, amie de l'artiste qui venait apprendre le dessin chez son père. Le journal qui relève du genre autobiographique constitue alors une sorte de décentrement. En effet, par ce récit se faisant par le truchement d'un regard et d'une sensibilité, ce n'est pas la vie de la jeune fille qui en est l'objet, mais elle recense ses premières rencontres avec Antoine Watteau, puis ses visites et les nouvelles qui lui viennent de Paris où il mène une carrière pleine de succès. Qu'il s'agisse d'informations obtenues par ouï-dire, de lettres, parfois destinées à d'autre et dont on lui a fait la lecture, la jeune fille se voit investie du rôle de biographe, composant un récit à partir des documents dont elle dispose.

Ainsi, la jeune fille pourrait apparaître, par un procédé métatextuel, comme la représentante de Pater lui-même et de son projet littéraire qui fait de l'espace de l'écriture le lieu d'un fantasme de l'imagination, qui puise dans sa propre subjectivité pour concevoir une existence nouvelle. La jeune fille, qui vit par procuration la réussite du peintre, s'exclame au moment de voir son frère partir pour embrasser la même vocation: "With how small a part of my whole life shall I be really living at Valenciennes!» (IP : 18). En réalité, si le journal laisse plus de place à d'autres personnages qu'au récit proprement autobiographique, c'est en vertu de la coïncidence des deux qui se fait dans l'hagiographie de l'artiste flamand. On retrouve ici un écho de 
la situation même de Pater, qui, retiré du monde n'en est pas moins son meilleur observateur, de telle sorte que ce mouvement de détachement, loin d'invalider le regard qu'il porte, semble la condition de son acuité : «It is the mood of the cloister taking a new direction, and winning so a later space of life it never anticipated " (Pater $1868:$ 216).

Ainsi, ce ne serait que par l'entremise de l'art que peut se révéler l'âme. En effet, loin de se contenter d'une restitution factuelle, la jeune fille tente de percer le mystère d'un homme taciturne et d'un génie qui reste distant et silencieux. Les pages de son journal sont ainsi ponctuées d'essais d'interprétation, d'hypothèses, par lesquels elle tente de déchiffrer sans en avoir aucune certitude le tempérament et les états d'âme du peintre. Par un effort d'imagination, elle se représente Antoine Watteau lui-même, au point de le croire véritablement présent devant ses yeux $^{23}$. Or, l'éloignement, qui n'est pas seulement géographique, mais le fait de sa fréquentation de cercles radicalement différents, lui fait pressentir et regretter sa capacité à se le représenter comme s'il était devant elle :

Certainly, great heights of achievement would seem to lie before him; access to regions whither one may find it increasingly hard to follow him even in imagination, and figure to one's self after what manner his life moves therein. (IP :

15)

Le peintre, qui est à l'initiative d'un nouveau style artistique dont le journal confesse que l'imagination demeure incapable de le concevoir ${ }^{24}$, revient à Valenciennes pour décorer la maison de ses anciens amis. Alors, devant les médaillons des Quatre Saisons qui ornent le grand salon, l'art devient l'instrument d'une révélation psychologique pour la jeune fille, capable à présent de saisir la personnalité d'Antoine Watteau : «I can understand through this, at last, what it is he enjoys, what he selects by preference, from all that various world we pass our lives in » (IP : 23).

Or, le peintre affichant quant à lui une certaine lassitude ${ }^{25}$ pourrait ne pas vivre la vie à laquelle rêve la jeune fille sur les pages de son journal : il « n'a certainement que peu de goût pour ses propres œuvres, - ces œuvres que, pour ma part, j'ai une telle soif de contempler " (IP : 19). De manière plus significative encore, croyant voir Watteau luimême en apercevant travailler son frère, l'imagination en vient à confondre les deux hommes pour créer un unique substitut, désormais disponible pour l'adorer : "JeanBaptiste's work, in its nearness to his, may stand, for the future, as the central interest of my life. I bury myself in that » (IP : 26). Ainsi, ce n'est pas tant l'existence réelle du jeune peintre qui est l'enjeu du journal, mais sa biographie fictive qu'imagine la jeune fille comme si, au fur et à mesure qu'elle écrit, elle vivait elle-même cette existence fantasmée ${ }^{26}$ :

One's journal, here in one's solitude, is of service at least in this, that it affords an escape for vain regrets, angers, impatience. One puts this and that angry spasm into it, and is delivered from it so. (IP : 39)

Le journal permet donc de manière privilégiée d'exposer le projet des Portraits, qui visent à montrer une existence en train de se faire, à en suivre l'itinéraire dans son élaboration même. C'est dans ce sens que l'on peut comprendre la mention récurrente du portrait de la jeune femme laissé inachevé : « My own poor likeness, begun so long ago, still remains unfinished on the easel » $(I P: 35)^{27}$. Un tel motif illustre la conception que développe Pater d'une vie qui n'est jamais accomplie, mais toujours ouverte et prête à être retouchée. Comme l'écrit Alexandre Gefen, il s'agirait alors de considérer que «le biographe s'interdit d'avoir le dernier mot» $(2012: 571)^{28}$. Il s'y exprime la 
terreur de l'œuvre achevée et close sur elle-même qui décourage toute forme de changement ou de transformation et bannit le mouvement par quoi se définit essentiellement la vie ${ }^{29}$.

Soulignons enfin l'importance de la médiation artistique. En effet, l'admiration portée au peintre est celle d'un art nouveau qui laisse transparaître une chose qui n'existe pas dans la réalité ordinaire. L'évocation de l'ennui et de l'insatisfaction face à l'existence trouve sa résolution dans la perspective de l'art qui offre des possibilités inouïes :

Besides that unreal, imaginary light upon these scenes, these persons, which is pure gift of his, there was a light, a poetry, in those persons and things themselves, close at hand we had not seen. He has enabled us to see it: we are so much the better-off thereby, and I, for one, the better. (IP : 32-33)

Dans l'article qu'il consacre à William Morris, «Aesthetic Poetry ", Pater évoque de la même façon une atmosphère poétique portant en elle des formes nouvelles ("The atmosphere on which its effect depends belongs to no simple form of poetry, no actual form of life ", Pater 1868 : 213), autrement dit il attribue à l'art la fonction d'enrichir notre vie d'éléments qui n'appartiennent pas à la réalité et nous permettent de vivre une existence plus intense.

\section{Le projet éthique des vies patériennes}

L'écriture des Portraits se trouve ainsi indissociable d'un enjeu éthique consistant à intensifier nos perceptions sensibles par la médiation de l'art («Our education becomes complete in proportion as our susceptibility to these impressions increases in depth and variety ", Pater 1873: Ix), afin de nous permettre de vivre notre existence pleinement. La vie ordinaire, soumise à l'habitude ${ }^{30}$ et aux catégories abstraites du langage ${ }^{31}$, serait appauvrie par l'ensemble de nos automatismes au point que la majeure partie de notre existence demeurerait inconsciente. L'art, avant même de rehausser les instants de notre vie, serait le moyen par lequel il serait permis de revenir sur l'ensemble des moments inaperçus de notre vie, considérés comme insignifiants pour libérer les trésors qu'ils recèlent. La reprise artistique, et notamment le travail de réécriture permet de s'apercevoir de l'épaisseur de la vie sensible et des possibles qu'elle contient.

La sensibilité humaine exigerait ainsi d'être sollicitée par des décors riches dans la vie quotidienne, aussi bien que par des formes imaginaires que produit l'art. En effet, la capacité à éprouver des sensations, à être affecté par l'environnement qui nous entoure et en saisir les moindres détails, doit être formée par l'exercice même de la sensibilité, qui se trouve favorisée par son dialogue avec l'art. Au contraire, la société victorienne marquée par la révolution industrielle et avec elle, la standardisation de la production et des modes de vie, menace par son caractère uniforme et monotone de porter atteinte à la sensibilité, au point de la rendre si grossière qu'elle ne sera plus en mesure de jouir des subtilités et des infimes variations que lui offre la réalité. En raison d'une sorte d'influence morale exercée par les objets qui composent notre monde quotidien, le manque d'attention dans leur choix ou la médiocrité de leur facture peut avoir pour conséquence de détériorer notre sensibilité alors que l'art aurait la vertu de « réveiller nos sens endormis » (Morris 1887 : 104). L'esthétique de Pater valorise ainsi l'art, non pas comme un monde en rupture avec la réalité mais comme un lieu privilégié dans lequel l'homme peut étendre la gamme de ses sensations, comme s'il affutait les 
instruments de ses sens, lui permettant ainsi de percevoir avec une plus grande acuité la vie ordinaire.

31 La conception de l'existence que développe Pater s'ancre dans une intuition précoce de la finitude humaine et dans un mouvement de révolte qui consiste à vouloir repousser autant que possible les limites qui définissent la vie humaine. S'il ne nous est pas permis de prolonger le nombre d'années de notre vie, il nous sera possible d'approcher une forme d'immortalité qui ne consiste pas dans un supplément de temps, mais dans l'intensité et l'approfondissement de nos sensations qui seraient le lieu d'une éternité de l'instant, qui déjoue leur fixation morbide ${ }^{32}$ par une attention fine à ce que les exigences de la vie quotidienne rendent invisible. Or, en demeurant inattentifs à ces détails, nous vivons la majorité de notre existence en pure perte. Le rôle de l'art sera alors de revenir sur chacun de ces instants pour leur restituer leur valeur et ainsi rendre à l'existence son intégrité. Le projet des vies s'inscrit donc d'emblée dans une conscience aiguë de la mort, autrement dit «attention inquiète à la temporalité humaine " (Gefen 2014: 11) qui fait l'étoffe de l'existence humaine et tout en lui donnant sa valeur oblige à répondre à l'injonction éthique de donner forme à cette vie :

For our one chance lies in expanding that interval, in getting as many pulsations as possible into the given time. Great passions may give us this quickened sense of life, ecstasy and sorrow of love, the various forms of enthusiastic activity, disinterested or otherwise, which come naturally to many of us. Only be sure it is passion-that it does yield you this fruit of a quickened, multiplied consciousness. Of such wisdom, the poetic passion, the desire of beauty, the love of art for its own sake, has most. For art comes to you proposing frankly to give the highest quality to your moments as they pass, and simply for thoses those moments' sake. (Pater $1873: 238-239$ )

L'existence ne saurait s'accomplir pleinement qu'en assumant cette part imaginaire, et la vie à laquelle nous contraint la société moderne n'offrirait aux yeux de Pater que de rares occasions de réjouissance ou de satisfaction, alors que l'art nous permettrait au contraire d'atteindre une qualité d'expérience renouvelée, et de repousser les bornes d'une vie individuelle finie: qu'il s'agisse des limites temporelles imposées à la vie humaine, aussi bien que les bornes de l'expérience propre, l'art offrirait un réservoir de virtualités qu'elle emprunte à l'ensemble de l'humanité dispersée dans l'espace et dans le temps, et qui seraient disponibles comme des «histoires particulières pouvant accueillir notre rêve universel d'habiter, le temps de la lecture d'autres existences que la nôtre" (Gefen 2014 : 11-12). Cet enrichissement que la vie trouve dans l'art, il l'exprime en opposant à la morne vie des hommes, l'existence héroïque des artistes qui se sont affranchis d'une telle monotonie: "people were prosaic, and their lives threadbare: - all but himself and organist Max, perhaps, and Fritz the treble-singer » (IP : 135).

33 L'art devient le lieu de méditation privilégié de cette question dans la mesure où la vie apporte sa part de sensations, mais réclame le secours de l'art pour ouvrir la possibilité de sensations nouvelles et inouïes par la réflexion et la reprise des expériences réelles. Germain d'Hangest peut ainsi parler d'une « une philosophie de l'excitation » (Hangest 1961 : 103) pour désigner cette valorisation de l'expérience autotélique, attentive aux sensations en vue d'une vie intense et donc réussie, et qui implique, par le secours de l'art, un enjeu éthique qui trouve dans l'esthétique le modèle d'une façon d'être ou d'une manière de vivre. Les Portraits imaginaires de Pater montrent ainsi à quel point la trame de notre vie est tissée d'imaginaire, empruntant à la fiction des formes d'existence dans lesquelles elles se projettent, ou faisant du rêve ou du désir des 
virtualités qui, si elles n'appartiennent pas à la réalité objective et avérée par les faits, ne peuvent être considérées comme " une sorte d'existence faible, balbutiée en dessous du seuil intègre de l'être » (Souriau $1943:$ 893). Or, ces « êtres de fictions » (Ibid. : 131), s'ils n'ont pas la consistance des corps, ne sont pas pour autant dénués d'existence, mais ils relèvent plutôt d'un mode d'existence «à base de désir, ou de souci, ou de crainte ou d'espérance, aussi bien que de fantaisie et de divertissement " (Ibid. : 133). Puisque "chaque mode est à soi seul un art d'exister " (Ibid.: 111), alors, cette classe existentielle des objets virtuels n'est pas seulement un univers irréel de fiction mais un monde qui en retour influence nos manières d'être ${ }^{33}$.

En ce sens, il annonce le projet proustien de la Recherche ${ }^{34}$, aussi bien dans la volonté de ressaisir une vie par un exercice d'écriture, que dans la conviction que le travail littéraire ne ferait pas que fixer une série d'événements, mais qu'elle rend possible de vivre pleinement ce qui demeurait simplement latent et que l'effort de reprise permet de déployer entièrement. Notons, en effet, que dans le premier de ses Portraits imaginaires, "L'enfant dans la maison", l'élément biographique, et même autobiographique, apparaît de la manière la plus évidente au point que Germain d'Hangest peut affirmer qu'il s'agit d'un «reflet poétique, un peu grandi par la mémoire » (Hangest 1961, 1:17) et qui constitue le récit programmatique de toutes les œuvres de Pater :

Dans ses romans et dans plusieurs de ses contes, en effet, il semble que ce soit

l'atmosphère essentielle, sinon la réalité de ce décor qu'il cherche à recréer, comme

si, mû par un besoin bien plus profond que la conscience, il lui fallait à toute force

retrouver le chemin de la maison perdue. (Hangest 1961, 1:17)

L'idée même de cette réélaboration illustre la méthode de Pater qui sans cesse retourne au trésor de la mémoire pour en exhumer le matériau, fait de sensations abandonnées, qui seront le germe d'une nouvelle création ${ }^{35}$. Apparait alors le motif d'une renaissance au sens strict du terme, autrement dit la reprise sous une forme nouvelle d'un contenu antérieur ou d'une virtualité qui ne s'était pas encore déployée : «Pater n'a pas cessé, durant toute sa carrière, d'être hanté par le mythe de la réminiscence, de la vie perpétuelle et de ses cycles. L'idée de réincarnation, de vie antérieure, tient dans son œuvre une très grande place » (Hangest $1964: 486$ ). Les Portraits seraient donc pour Pater des moyens non seulement d'explorer différentes options éthiques et d'interroger la vie humaine comme un anatomiste de l'âme, comme si chacun d'eux ne faisait qu'approfondir et développer un trait de caractère, un événement, un souvenir du passé et auquel la littérature offre la possibilité de suivre le cours hypothétique comme une voie possible de l'existence n'ayant pas été retenue et donc actualisée, mais demeurant à l'état fantasmatique comme un halo des virtualités qui subsistent à leur stade embryonnaire dans l'individu :

On retrouve ainsi une des idées essentielles de la philosophie de Pater, à savoir la capacité re-créatrice d'une "mémoire poétique " (Hangest 1961, 1:19). À partir des souvenirs recueillis, elle libère par l'intermédiaire de l'art la puissance sensuelle de ces impressions diffuses qui ont marqué tout individu comme la réserve inépuisable de ses vies imaginaires, de ces virtualités inexplorées de l'existence dont l'expérience n'a laissé que la trace en guise de suggestion. En ce sens, les Portraits supportent un « désir d'existence toujours ouvert, tout en rendant au lecteur un espace où projeter ses propres possibles, souvent hors du monde » (Gefen 2015 : 15). Ce n'est qu'en lui-même qu'il trouve une source de création possible, et qui prend la forme de différents 
masques, construisant une personnalité poétique, semblable à un "palimpseste indéchiffrable à force d'être saturé de réinscriptions » (Regard 2002 : 97) :

It is hardly an exaggeration to say that in Plato, in spite of his wonderful savour of literary freshness, there is nothing absolutely new: or rather, as in many other very original products of human genius, the seemingly new is old also, a palimpsest, a tapestry of which the actual threads have served before, or like the animal frame itself, every particle of which has already lived and died many times over. (Pater $1893: 8)$ promotion d'une posture contemplative, d'une attention aux détails et d'un souci de soi, s'inscrit en contradiction avec l'idéologie dominante de la société victorienne. Matthew Arnold, auteur de Culture and Anarchy, défendra la nécessité de pratiques inutiles, n'ayant de fin qu'en elle-même (« a desire after the things of the mind simply for their own sakes and for the pleasure of seeing them as they are ", Arnold 1869:6) et non en vue d'un bénéfice matériel. Le principe d'utilité qui structure la société britannique industrielle conduit à rendre les rapports humains et la vie elle-même mécanique ${ }^{38}$. Par les figures qu'il convoque et à travers lesquels il construit sa propre identité, Pater semble ménager un espace de résistance permettant de résister au paradigme instrumental largement diffusé, pour lui substituer une conception plus 
riche de l'existence et se proposer un art suprême qui serait cet "art plus difficile encore de vivre » (Schiller $1795: 221)$ :

Il est permis de trouver là, explicite dès à présent quoique furtive, une conception du style comme une conception de la conduite ; éthique et esthétique déjà ne font qu'un, puisque la vie dans son ensemble, aspire aux conditions de l'art, et que la même perfection musicale doit ordonner, aussi bien que les œuvres, les existences mêmes. (Hangest 1961, 1: 70)

Dans les divers textes que consacre Michel Foucault à "l'esthétique de l'existence ", il en propose des figures qui viennent scander une généalogie des pratiques de soi, depuis les philosophies grecques ascétiques jusqu'à la société contemporaine ${ }^{39}$. Dans cette histoire, il s'attarde sur la Renaissance, y reconnaissant comme Pater un moment où l'art avait pu déterminer des façons de vivre. Au fil de cette chronologie, il met ensuite l'accent sur le dandysme du XIX ${ }^{e}$ siècle, dont Pater fut une des figures principales et surtout un théoricien important. Ainsi, dans une société à la morale rigide et marquée par des impératifs de rentabilité, Pater s'inquiète de la possible disparition du beau et de l'art, et à travers eux d'une existence pleinement humaine, riche de sensations, consciente de sa vulnérabilité et toujours animée de désir. Les vies imaginaires sont alors autant de destins singuliers qui permettent d'explorer des virtualités de l'existence, et d'en esquisser des orientations possibles, rares et originales. Il s'agit bien, contre la morale tout à la fois contraignante et uniformisante, d'affirmer la singularité d'une manière d'être, d'une personnalité qui manifeste par son existence même un certain art de vivre et participe ainsi d'une éthique de la création de soi comme individu.

\section{BIBLIOGRAPHIE}

Arnold, Matthew, [1869] 1903, Culture and Anarchy. An Essay in Political and Social Criticism, Complete Works VI, Londres, Macmillan and Co.

Boyer-Weinmann, Martine, 2004, « La biographie d'écrivain : enjeux, projets, contrats. Cartographie exploratoire d'un geste critique », Poétique, nº 139, p. 299-314.

Buisine, Alain, 1991, « Biofictions », Revue des Sciences Humaines, nº 224, p. 7-13.

Ellmann, Richard, [1969] 1994, Oscar Wilde, trad. M. Tadié et P. Delamare, Paris, Gallimard.

Foucault, Michel, [1984] 2001, « À propos de la généalogie de l'éthique : un aperçu du travail en cours ", dans Dits et Écrits II, Paris, Éditions Gallimard, texte ${ }^{\circ} 344$.

Gefen, Alexandre, 2002, « Vie imaginaire et poétique du roman au XIX siècle : la Notice biographique de Louis Lambert », Littérature, $n^{\circ}$ 128, p. 3-25.

Gefen, Alexandre, 2012, «Au pluriel du singulier : la fiction biographique », Critique, vol. 781-782, $\mathrm{n}^{\circ} 6$, p. 565-575.

Gefen, Alexandre, 2014, « Préface », Vies imaginaires. De Plutarque à Michon, Paris, Gallimard. 
Gefen, Alexandre, 2015, « Introduction », Inventer une vie. La fabrique littéraire de l'individu, Bruxelles, Les impressions nouvelles.

Korthals Altes, Liesbeth, 1999a, «Le tournant éthique dans la théorie littéraire : impasse ou ouverture », Études littéraires, vol. 31, n 3, p. 39-56.

Korthals Altes, Liesbeth, 1999b, « Présentation : Éthique et littérature », Études littéraires, vol. 31, $\mathrm{n}^{\circ}$ 3, p. 7-13.

Laugier, Sandra, 2006, « Présentation », dans S. Laugier (dir.), Éthique, littérature, vie humaine, Paris, PUF, p. 4-18.

Macé, Marielle, 2011, Façons de lire, manières d'être, Paris, Gallimard.

Morris, William, [1877] 2013, « Les arts mineurs » (Conférence donnée devant le Trades Guild of Learning), dans Comment nous vivons, comment nous pourrions vivre, trad. F. Guévremont, Paris, Payot et Rivages.

Proust, Marcel, [1927] 1989, Le temps retrouvé, À la recherche du temps perdu IV, Paris, Gallimard. Regard, Frédéric, 2002, "L'Éthique du biographique. Réflexions sur une tradition britannique », Littérature, $\mathrm{n}^{\circ} 128$, p. 80-92.

Schiller, Friedrich, [1795] 1992, Lettres sur l'éducation esthétique, trad. R. Leroux, Paris, Aubier. Schwob, Marcel, [1896] 2002, «Préface » aux Vies imaginaires, dans Euvres, Paris, Phébus. Snauwaert, Maïté et Caumartin, Anne, 2010, « Présentation : Éthique, Littérature, Expérience », Études françaises, vol. 46, $\mathrm{n}^{\circ}$ 1, p. 5-14.

\section{Corpus}

\section{Euvres de Pater}

Pater, Walter, [1887] 1910, Complete Works IV: Imaginary Portraits, Londres, Macmillan and Co.

Pater, Walter, [1873] 1912, Complete Works I: The Renaissance. Studies in Art and Poetry, Londres, Macmillan and Co.

Pater, Walter, [1889] 1910, «Style », Complete Works V: Appreciations with an Essay on Style, Londres, Macmillan and Co.

Pater, Walter, [1893] 1910, Complete Works VII: Plato and Platonism, Londres, Macmillan and Co.

Pater, Walter, [1864] 1910, « Diaphanéité », Complete Works VIII: Miscellanies Studies, Londres, Macmillan and Co.

Pater, Walter, [1868] 1889, « Aestheric Poetry », Apprciations with an Essay on Style, Londres, Macmillan and Co.

Pater, Walter, 1970, Letters of Walter Pater, éd. Lawrence Evans, Oxford, Clarendon Press.

\section{Littérature secondaire à propos de Pater}

Beckson, Karl et Munro, John, Autumn, 1970, « Symons, Browning, and the Development of the Modern Aesthetic », Studies in English Literature, 1500-1900, vol. 10, n 4, p. 687-699. 
Blinderman, Charles S., 1982, « Huxley, Pater, and Protoplasm », Journal of the History of Ideas, vol. $43, \mathrm{n}^{\circ} 3$, p. $477-486$.

Buckler, William, 1978, « "Marius the Epicurean”: Beyond Victorianism », Victorian Poetry, vol. 16, $\mathrm{n}^{\circ} 1 / 2$, p. 147-166.

Buuren, Maarten van, 2004, « Le dilettantisme, style de vie », Poétique, nº 137, p. 53-71.

Candido, Anne Marie, 1993, « Biography and the Objective Fallacy: Pater's Experiment in “A Prince of Court Painters" ", Biography, vol. 16, n² 2, p. 147-160.

Child, Ruth C., 1969, The Aesthetic of Walter Pater, New York, Octagon Books.

Coste, Bénédicte, 2003, « Introduction » à Walter Pater, Textes esthétiques, Nîmes, Théétète éditions.

Dowling, Linda, 1988, « Walter Pater and Archaeology: The Reconciliation with Earth », Victorian Studies, vol. 31, $\mathrm{n}^{\circ} 2$, p. 209-231.

Hangest (D'), Germain d', 1961, Walter Pater. L'homme et l'œuvre, Paris, Didier.

Hangest (D'), Germain d', 1964, « Un grand humaniste de la fin du dix-neuvième siècle : Walter Pater et la Grèce », Bulletin de l'Association Guillaume Budé: Lettres d'humanité, nº 23, p. 479-493.

Johnson, Lionel, 1911, Post liminium. Essays and critical papers, éd. T. Whittermore, Londres, Elkin Mathews.

Lambert-Charbonnier, Martine, 2004, Walter Pater et les « Portraits Imaginaires ». Miroirs de la culture et images de soi, Paris, L'Harmattan.

Leyris, Pierre, 1992, « Préface » à Walter Pater, L'enfant dans la maison, Paris, José Corti.

Monsman, Gerald, 1977, Walter Pater, Boston, G. K. Hall \& Co.

Monsman, Gerald, 1980, Walter Pater's Art of Autobiography, New Haven, Yale University Press.

More, Paul Elmer, 1913, The Drift of Romanticism: Shelburne Essays, Eighth Series, Boston, Houghton Mifflin Company.

Neel, Philippe, 1930, « Avant-propos » à Walter Pater, Portraits imaginaires, Paris, Christian Bourgeois éditeur.

Praz, Mario, [1972] 1990, Le Pacte avec le serpent, vol. 2, Paris, Christian Bourgois.

Praz, Mario, [1930] 1998, La Chair, la Mort et le Diable : le romantisme noir, Paris, Gallimard.

Roger-Cornaz, Frédéric, 1917, « Introduction » à Walter Pater, La Renaissance, Paris, Librairie Payot et Cie.

Salha, Agathe, 2006, « Discours critique et fiction biographique dans les Portraits imaginaires de Pater et les Vies imaginaires de Schwob », Recherches \& Travaux, nº 68, p. 29-39.

Symons, Arthur, 1873, «Introduction », Walter Pater, The Renaissance, New York, The Modern Library.

Symons, Arthur, 1904, Studies in Prose and Verse, Londres, J.-M. Dent \& Co.

Wilde, Oscar, [1887] 1905, Complete Works IX: Review, éd. R. Ross, Boston, Wyman-Fogg Company. Williams, Carolyn, 1989, Transfigured World: Walter Pater's Aesthetic Historicism, Ithaca, Cornell University Press. 


\section{NOTES}

1. À propos de la véracité de l'anecdote, cf. Symons $(1873: \mathrm{xxI}):$ «I once asked Pater if his family was really connected with that of the painter, Jean-Baptiste Pater. He said: "I think so, I believe so, I always say so." The relationship has never been verified, but one would like to believe it; to find something lineally Dutch in the English writer. "

2. Désormais abrégé en en IP dans les références à l'œuvre de Pater.

3. La conclusion de La Renaissance insiste sur ce caractère évanescent d'une réalité se transformant en permanence et dont l'analyse ne pourrait que la réduire à ses éléments essentiels : " phosphorus and lime and delicate fibres » (Pater [1873] $1912: 233$ ).

4. Notons que cette reprise moderne du thème héraclitéen d'une réalité mouvante n'est pas sans lien avec une résurgence de l'atomisme dans la pensée moderne, que l'on retrouve également chez Lucrèce, dont H. A. J. Muneo offre une traduction du De natura rerum en 1864. Cf. Williams (1989: 15) : «Even more than Pater's tacit claim to mastery of the tradition is the hint that "modern" thought is not so thoroughly new, but is in a many ways only a "modernization" of the classical tradition. The epigraph quietly shows, to those who read Greek, that Pater believes the threat of "modern" thought to be an ancient, a persistent, even a traditionnal threat. For the present study, this epigraph will serve as a brief introduction to Pater's habit of finding "mythic" recapitulations in the history of thought, since here the latest findings of science and philosophy suggest to him an analogue in Heraclitus. »

5. Moins qu'une influence directe de Darwin, il s'agit surtout d'un écho des lectures de Thomas H. Huxley. Cf. Blinderman (1982: 483) : «For Pater, the protoplasmic whirlpool illustrates the fundamental dynamics of our life, especially its turbulence and transiency. Alert to the implications of such scientific finds, we will sharpen our perception so that we could use the interval remaining to us in the best way, that is, by burning with a hard gem-like flame.»

6. Des héros des Portraits imaginaires aux artistes de La Renaissance et même jusqu'aux penseurs de la philosophie grecque des conférences sur Platon et le platonisme, Mario Praz constate que « tous les personnages de Pater appartiennent à des époques de transition ou - pourrait-on dire - sont nés hors du temps: ils doivent faire face à des circonstances qu'ils ne sont pas à même d'affronter, ou ils errent dans des méandres de possibilités dans lesquelles ils dispersent leurs énergies» (Praz 1990 : 40).

7. «De caractère timide, rêveur et méditatif, il ne joua point comme jouent ordinairement les enfants; mais dans une pièce inutilisée au fond de la maison, il faisait semblant d'être prêtre, s'affublait d'une robe de chambre en guise de surplis, et du haut d'une chaire fictive, prêchait des sermons à sa mère et à la tante Bessie émerveillées. De ce jeu-là, il ne se lassait jamais, et même il insistait pour le reprendre tous les jours » (Hangest 1961, $1: 16-17)$.

8. Neel (1930: X-XII) : «Il mena une existence paisible et monotone. [...] Il passa presque toute sa vie dans l'antique cité, consacrant ses forces de moine laïque à ses cours et à ses livres. [...] La danse de Pater est lente et grave, et s'inscrit dans un cercle étroit comme celui d'une prison. Prison aimable au surplus que celle de la docte cité, de ses antiques collèges, de ses bibliothèques, des brumes perlées qui montent des prairies de l'Isis. Pater vit d'Oxford et de souvenirs. » Cette description d'une vie recluse se retrouve également sous la plume de Mario Praz : «L'existence de Pater s'écoule monotone dans les pièces soignées mais sobres de Brasenose College et ensuite de Londres, dans des contacts sociaux disciplinés par son indéfectible sens du rituel » (Praz 1998 : 295).

9. D'autres passages témoignent également de cette capacité à percevoir la réalité avec plus de finesse en raison même d'une forme de retrait: «If I understand anything of these matters, Antony Watteau paints that delicate life of Paris so excellently, with so much spirit, partly because, after all, he looks down upon it or despises it. " (IP : 26-27). Le thème du mépris des mondanités court tout le long du portrait, de même que l'idée d'une mise à distance nécessaire et 
bénéfique à la création d'une œuvre comme si la représentation exigeait que l'artiste ne soit pas impliqué : "And just where the crowd was busiest young Antony was found, hoisted into one of those empty niches of the old Hotel de Ville, sketching the scene to the life.» (5); «He goes but rarely into the society whose manners he has to re-produce » (28).

10. Child (1969:2) : "When this epoch of world change is past-as epochs do pass-Pater will still be found dealing graciously with the central human problem, how to live. »

11. Laugier (2006: 4): «Le travail de la critique (littéraire) sera alors la recherche d'une définition de l'éducation donnée par l'œuvre, non pas au sens d'une édification ou de la transmission d'un contenu défini, mais au sens où cette œuvre elle-même apprend à son lecteur à la considérer, à vivre sa propre aventure (morale) de lecteur. Il faut en quelque sorte arriver à définir dans ce cadre une morale non moralisatrice. [...] L'œuvre littéraire n'a pas à donner des jugements ou à conduire (explicitement ou subrepticement) le lecteur à une conclusion morale. Mais elle est morale dans la mesure où elle transforme le lecteur, son rapport à son expérience elle fait partie en quelque sorte de sa vie.»

12. Anne Marie Candido insiste notamment sur la présence d'un schéma biographique ("the conventional birth-to-death pattern ») déjà présent dans les essais : "Walter Pater was among the most radical aesthetic reformers of his time, not only in his theory but particularly in his approach to biographical writing. [...] Pater never refers to the portraits contained in the work as biographies but rather as "studies," or "essays," it is obvious enough that with the exception of the first essay these "studies" were written in the form of brief lives " (Candido 1993 : 147-148).

13. Pater (1889: 9) : « Your historian, for instance, with absolutely truthful intention, amid the multitude of facts presented to him must needs select, and in selecting assert something of his own humour, something that comes not of the world without but of a vision within. »

14. On peut trouver la confirmation de cette idée dans les écrits que consacre Pater à la question du style : « The transcript of his sense of fact rather than the fact, as being preferable, pleasanter, more beautiful to the writer himself. [...] Literary art, that is, like all art which is in any way imitative or reproductive of fact-form, or colour, or incident-is the representation of such fact as connected with soul, of a specific personality, in its preferences, its volition and power " (Pater 1889 : 14-15).

15. Schwob (1896: 511). Cf. Salha (2006: 29) : «Autrement dit, et pour reprendre les termes de la préface des Vies imaginaires, les Portraits de Pater illustreraient plutôt le point de vue généralisant de l'histoire, alors que la démarche biographique de Schwob serait axée sur la recherche du particulier et de l'individuel.»

16. Symons (1904: 67) : "What he has done is to give a concrete form to abstract ideas; to represent certain types of character, to trace certain developments in the picturesque form of narrative; to which, indeed, the term portrait is very happily applied; for the method is that of a very patient and elaborate brushwork, in which the touches that go to form the likeness are so fine that it is difficult to see quite their individual value, until, the end being reached, the whole picture starts out before you. "

17. C'est bien aussi l'interprétation qu'en fera son élève Oscar Wilde, prolongeant avec la conception d'une critique-artiste, la possibilité d'en passer par la fiction et par des formes littéraires originales pour formuler des idées. Cf. Wilde $(1887: 172)$ : « For these Imaginary or, as we should prefer to call them, Imaginative Portraits of his, form a series of philosophic studies in which the philosophy is tempered by personality, and the thought shown under varying conditions of mood and manner, the very permanence of each principle gaining something through the change and colour of the life through which it finds expression. " Ce faisant, il emprunte à Platon, selon sa propre interprétation, l'idée que toute connaissance n'est possible que par l'entremise d'une personnalité, dont le Dialogue donnerait la forme, et qui avait été déjà largement mis en valeur par Pater lui-même dans ses études consacrées à la philosophie grecque (Pater 1893 : 102). 
18. Si Pater s'inspire de Sainte-Beuve et reprend pour une bonne part son Port-Royal pour son texte sur Pascal (1895, Contemporary Review), il rompt également avec les Portraits littéraires aussi bien dans ses essais que dans ses œuvres de fiction. Cf. Coste (2003: 8) : «Pater traverse les divisions et les chapelles avec l'aisance que lui donne sa conception rigoureuse du sujet, à travers le genre du portrait littéraire qu'il n'a pas inventé mais repris à Sainte-Beuve, encore qu'il lui ait fait subir quelques transformations [...] L'homme et l'œuvre à la Sainte-Beuve c'est l'homme moins l'œuvre, l'anecdote plutôt que l'accueil et l'étude de ce qui se manifeste dans la poétique. Chez Pater, rien de tout cela et, dès les premiers portraits de La Renaissance (Pic de la Mirandole, Léonard, Michel-Ange), le ton est donné, le modèle français abandonné. Loin d'expliquer l'œuvre par l'homme, Pater fait de la représentation littéraire du sujet le lieu de rassemblement des impressions suscitées par l'œuvre, l'écran indispensable à la relation esthétique. Entre l'œuvre et le spectateur esthète, le portrait est un lieu de négociation, de recueil des émotions élevées à la dignité littéraire. »

19. En effet, Mario Praz voit dans les Portraits imaginaires, un prolongement naturel du projet entrepris par Pater avec La Renaissance, mais lui offrant un espace de plus grande liberté par le biais de la fiction. Cf. Praz (1990: 42) : "Le caractère mélangé, de critique et d'imagination, si typique de La Renaissance, laissait prévoir le développement ultérieur: les données historiques n'étaient pour le critique-artiste qu'un tremplin pour recréer ses fantaisies passionnées, pour ses audacieuses insinuations d'un frémissement très moderne sur des personnes et des choses du passé qui, à la lumière d'une froide critique, impliquaient mal cette séduisante liberté. À la longue, le personnage historique se révélait incapable de supporter le contenu dont l'auteur voulait le charger et auquel il tenait davantage. Il devenait climat culturel, problème psychologique : climat et problème qui reflétaient ceux de Pater et de son temps. C'est d'ailleurs ce qui se passe toujours lorsqu'on écrit une histoire, mais ici le cas était extrême. Par conséquent, Pater s'évada de l'essai critique dans le "portrait imaginaire".» On retrouve dans son introduction aux Portraits la même analyse chez Leyris: "La forme littéraire de l'essai, cependant, avait ses limites et ne permettait pas à Pater de s'exprimer tout entier» (Leyris 1992 : 9-10).

20. "Among old Watteau's workpeople came his son, the genius, my father's godson and namesake, a dark-haired youth » (IP: 5 ).

21. On le retrouve également dans la figure du narrateur de «Denys l'Auxerrois »: "With this fancy in my mind, by the help of certain notes, which lay in the priest's curious library, upon the history of the works at the cathedral during the period of its finishing, and in repeated examination of the old tapestried designs, the story shaped itself at last » (IP : 54).

22. "Of the French town, properly so called, in which the products of successive ages, not without lively touches of the present, are blended together harmoniously, with a beauty specific » (IP : 48) ; " examine its relics, old glass and the like, of the Renaissance or the Middle Age » (IP : $51)$; " every touch of art or of time on its old building is defined in clear grey " (IP: 52). On retrouve ici la conception qu'emprunte Pater à la biologie d'Huxley, au matérialisme héraclitéen et aux travaux de l'archéologie moderne, à savoir l'idée de formes primitives qui constitueraient l'essence de la trame d'une réalité renaissant toujours à elle-même à partir de ses propres traces. Cf. Dowling (1988 : 220) : «Pater's keen sense of the material origin and basis of culture persists throughout his œuvre, and finds expression in his recurrent portrayal of the earth as the actual medium of biological as well as cultural rebirth. Hence the repeated images in his works of buried relics, opened graves, and beautiful corpses: these are the metaphors of material transfiguration."

23. «Ah! how I delight myself, in fancy at least, in those beautiful gardens, freer and trimmed less stiffly than those of other royal houses. Methinks I see him there, when his long summerday's work is over, enjoying the cool shade of the stately, broad-foliaged trees, each of 
which is a great courtier, though it has its way almost as if it belonged to that open and unbuilt country beyond, over which the sun is sinking. » (IP : 10)

24. « And Antony's new manner? I am unable even to divine it-to conceive the trick and effect of it-at all $»(I P: 16)$.

25. IP (11) : «There is an air of seemly thought-le bel serieux-about him, which makes me think of one of those grave old Dutch statesmen in their youth, such as that famous William the Silent. »; «It is rather as if, with all that success, life and its daily social routine were somewhat of a burden to him» (IP : 20).

26. «With myself, how to get through time becomes sometimes the question,-unavoidably; though it strikes me as a thing unspeakably sad in a life so short as ours » (IP : 25). Nous pouvons renvoyer également à un autre extrait : «I am just returned from early Mass. I lingered long after the office was ended, watching, pondering how in the world one could help a small bird which had flown into the church but could find no way out again. I suspect it will remain there, fluttering round and round distractedly, far up under the arched roof, till it dies exhausted. I seem to have heard of a writer who likened man's life to a bird passing just once only, on some winter night, from window to window, across a cheerfully-lighted hall. The bird, taken captive by the ill-luck of a moment, re-tracing its issueless circle till it expires within the close vaulting of that great stone church:-human life may be like that bird too!» (IP: 14-15). On retrouve cette méditation sur la finitude humaine qui faisait déjà l'objet de la conclusion de La Renaissance.

27. On en trouve d'autres formulations : «My own portrait remains unfinished at his sudden departure. » (24); «Jean-Baptiste, who will be heir to his unfinished work, and take up many of his pictures where he has left them » (42).

28. Alexis Tadié insiste lui aussi sur l'enjeu d'une éthique du biographique qui consisterait, non pas à privilégier la trame d'une vie finie, mais de rendre compte par le récit de ses virtualités. «C'est dans cette distance incommensurable, dans la fragilité inhérente au genre, dans cette hésitation sur l'interprétation que l'on peut donner de son objet en même temps que dans l'obligation de le faire, qu'intervient la littérature. Elle permet d'exprimer "l'essaim de possibilités" de l'être plutôt que de se réfugier derrière un récit trop uniforme " (Tadié 2012 : 562).

29. En ce sens on pourra évoquer la qualification de divers critiques, à commencer par Wilde, qui reconnaissent au style de Pater une dimension impressionniste, travaillant par touches successives, comme se méfiant de l'image directement formée. Cf. Wilde (1887 : 174) : «Mr. Pater is an intellectual impressionist. He does not weary us with any definite doctrine or seek to suit life to any formal creed. He is always looking for exquisite moments and, when he has found them, he analyses them with delicate and delightful art and then passes on, often to the opposite pole of thought or feeling, knowing that every mood has its own quality and charm and is justified by its mere existence. "

30. " It might even be said that our failure is to form habits: for, after all, habit is relative to a stereotyped world, and meantime it is only the roughness of the eye that makes any two persons, things, situations, seem alike " (Pater 1873 : 236-237).

31. "If we continue to dwell in thought on this world, not of objects in the solidity with which language invests them, but of impressions, unstable, flickering, inconsistent, which burn and are extinguished with our consciousness of them, it contracts still further: the whole scope of observation is dwarfed into the narrow chamber of the individual mind " (Pater $1873: 235$ ).

32. "What we have to do is to be for ever curiously testing new opinions and courting new impressions, never acquiescing in a facile orthodoxy » (Pater 1873 : 237).

33. "Chaque roman, chaque tableau même est à certains égards un microcosme [, et] de tous ces microcosmes se compose une sorte de grand cosmos littéraire et artistique, où certains personnages types ont une existence innombrable et pourtant essentielle et identique (songez à 
Don Juan). Donc, par un côté, ce monde tend à prendre une existence syndoxique, sociale, bien positive » (Souriau $1943: 132$ ).

34. On pense notamment à ce passage du Temps retrouvé : « La vraie vie, la vie enfin découverte et éclaircie, la seule vie par conséquent réellement vécue, c'est la littérature » (Proust 1927 : 474).

35. Mario Praz exprime cette idée d'une germination dans des voies multiples à partir d'un fond commun, ou d'une unique semence, en écrivant: "L'art de Pater est fondé sur quelques impressions indélébiles et approfondit mille nuances minutieusement observées : L'Enfant dans la maison nous donne la clé de Marius l'Epicurien et aussi de Sébastien Van Storck qui présente, dans un milieu différent, des thèmes analogues » (Praz 1990 : 50).

36. "Cette nécessité d'une construction rhétorique du soi est sans doute dictée par la conception que l'on se fait depuis longtemps en Angleterre de l'identité personnelle, identité qui entretient avec la mémoire comme avec l'imagination des rapports complexes. Entre le chapitre 27 de l' Essay Concerning Human Understanding de John Locke (1694) et le Livre I du Treatise on Human Nature de David Hume (1739), s'installe en effet un débat qui place la question de l'identité personnelle au cœur de la philosophie anglo-saxonne, dont la production biographique fonctionne alors comme l'un des prolongements littéraires. [...] On voit se dessiner ici un lien étroit avec le travail biographique, qui se donne également pour tâche de rassembler les événements d'une vie à l'intérieur de ce que Hume nommerait “a train of connected ideas", c'està-dire de ce que nous nommerions aujourd'hui un récit ou un "plot". Cette subtile connivence de la mémoire et de la fabulation, de l'archivage des faits bruts et de leur reconfiguration, se retrouve en effet dans la biographie littéraire, qui ne peut échapper dans sa recherche de l'identité de l'autre à cette nécessité d'une fictionnalisation de la vie » (Regard 2002 : 86).

37. «[...] those impressions of the individual mind to which, for each one of us, experience dwindles down, are in perpetual flight » (Pater 1873 : 235) ; «that continual vanishing away, that strange, perpetual weaving and unweaving of ourselves » (236).

38. " [...] it is clear that culture, instead of being the frivolous and useless thing which Mr. Bright, and Mr. Frederic Harrison, and many other Liberals are apt to call it, has a very important function to fulfill for mankind. And this function is particularly important in our modern world, of which the whole civilisation is, to a much greater degree than the civilisation of Greece and Rome, mechanical and external, and tends constantly to become more so » (Arnold 1869 : 13).

39. «Il s'agissait de faire de sa vie un objet de connaissance ou de tekhnê, un objet d'art. Nous avons à peine le souvenir de cette idée dans notre société, idée selon laquelle la principale œuvre d'art dont il faut se soucier, la zone majeure où l'on doit appliquer des valeurs esthétiques, c'est soi-même, sa propre vie, son existence. On retrouve cela à la Renaissance mais sous une forme différente, et encore dans le dandysme du XIX ${ }^{e}$ siècle, mais ce n'ont été que de brefs épisodes » (Foucault $1984:$ 1443).

\section{RÉSUMÉS}

La critique de Walter Pater témoigne, dans les études qu'il propose, d'une attention particulière à la personnalité des artistes, considérant que l'œuvre trouve son explication dans un tempérament particulier. Ainsi, la réflexion sur l'individu et la vie parcourt toute son œuvre, jusqu'aux écrits à proprement parler de fiction, et les Portraits Imaginaires. Le récit de la vie s'y déploie pleinement, comme autant d'existences virtuelles empruntées aux impressions de son 
auteur et lui permettant de défendre une véritable éthique fondée sur l'art: la brièveté de l'existence nous conduit à employer l'art comme un moyen d'approfondir nos sensations et d'explorer des vies imaginaires.

The personality of the artists is very important for Walter Pater in his critical works, because he considers that a singular temperament can provide an explanation of the piece of art. For this reason, the reflection about the individual is present in most of his texts, and especially in his fictional writings, the Imaginary Portraits. The narrative account of oneself is here treated like some virtual lives based on Pater's own impressions. They show an ethical point of view where art could help us to extend our short life by going deeper into our own sensations.

INDEX

Mots-clés : biographie, identité, personnalité, imagination, art, Pater (Walter)

Keywords : biography, identity, personnality, imagination, art, Pater (Walter)

\section{AUTEUR}

\section{ALEXANDRE BIES}

DCCE - Université de Nice Sophia Antipolis 